\title{
Interação genótipo-ambiente de soja convencional e transgênica resistente a glifosato, no Estado do Paraná
}

\author{
Wilmar Ferreira Lima(1), Antonio Eduardo Pípolo(2), José Ubirajara Vieira Moreira(2), \\ Claudio Guilherme Portela de Carvalho(2), Cássio Egídio Cavenaghi Prete ${ }^{(1)}$, Carlos Alberto Arrabal Arias(2), \\ Marcelo Fernandes de Oliveira(2), Geraldo Estevam de Souza ${ }^{(2)}$ e José Francisco Ferraz de Toledo ${ }^{(2)}$
}

\begin{abstract}
(1) Universidade Estadual de Londrina, Departamento de Agronomia, Caixa Postal 6.001, CEP 86050-990 Londrina, PR. E-mail: wilmar@sercomtel.com.br, cassio@uel.br(2)Embrapa Soja, Caixa Postal 231, CEP 86001-970 Londrina, PR.E-mail:pipolo@cnpso.embrapa.br, bmoreira@cnpso.embrapa.br, cportela@cnpso.embrapa.br, arias@cnpso.embrapa.br, marcelo@cnpso.embrapa.br, estevam@cnpso.embrapa.br, toledo@cnpso.embrapa.br
\end{abstract}

Resumo - Os objetivos deste trabalho foram: comparar a produtividade e a adaptação de genótipos de soja convencional e de soja transgênica resistente ao herbicida glifosato, de diferentes grupos de maturação, desenvolvidos pelo programa de melhoramento da Embrapa Soja para o Estado do Paraná; estudar a importância relativa dos efeitos de local, ano, cultivar e suas respectivas interações; e verificar a possibilidade de se estratificar o Estado em regiões mais homogêneas, para reduzir o número de locais nos ensaios de competição de linhagens. Foram utilizados dados de produtividade de grãos de ensaios regionais, no Estado do Paraná, entre 2001 e 2005. O delineamento experimental utilizado foi o de blocos ao acaso. A possibilidade de se realizar a estratificação do Estado em regiões mais homogêneas e de descarte de locais foi verificada pela significância da interação genótipo $\mathrm{x}$ ambiente entre locais. Não houve diferença significativa de produtividade entre a soja convencional e a transgênica, independentemente do grupo de maturação. O efeito de local foi mais importante que o efeito de ano, na composição dos ambientes. A estratificação do Estado do Paraná em regiões não trouxe vantagens, nos anos analisados, para os testes de linhagens; apenas os locais da região Sul mostraram algum grau de similaridade entre si.

Termos para indexação: Glycine max, adaptação em soja, estratificação ambiental, melhoramento de plantas, melhoramento de soja.

\section{Genotype x environment interaction of conventional and glyphosate-resistant genetically engineered soybean in Paraná State, Brazil}

\begin{abstract}
The objectives of this research were: to compare productivity and adaptation of conventional and glyphosate-resistant genetically engineered soybean genotypes, from different maturity groups, developed by the breeding program at Embrapa Soja for Paraná State, Brazil; to study the relative importance of the effects of location, year, cultivar and their respective interactions; and to verify the possibility of stratifying the State in more homogeneous regions, in order to reduce the number of locations for line competition experiments. Paraná State yield data from regional experiments carried out in randomized complete block design from 2001 to 2005 were used. The possibility of stratifying the State in homogeneous regions and discarding similar locations was checked by the significance of genotype $\mathrm{x}$ environment interaction among locations. Yield was not significantly different between genetically engineered and conventional soybean, regardless of the maturity group. The effect of location was more important than the effect of year in the environment composition. The stratification of Paraná State in regions for yield testing soybean lines brought neither significant nor consistent advantages for the evaluated years. Only locations within the South region showed some degree of similarity.
\end{abstract}

Index terms: Glycine max, adaptation in soybean, environmental stratification, plant breeding, soybean breeding.

\section{Introdução}

O Estado do Paraná produziu aproximadamente 12 milhões de toneladas de soja, em uma área de cerca de 4 milhões de ha, na safra de 2006/2007, tendo-se classificado como o segundo maior produtor com a segunda maior área de soja entre os estados brasileiros (Conab, 2007). Nessa safra, $47 \%$ das cultivares 
plantadas eram transgênicas, com genes de resistência ao herbicida glifosato (Paraná, 2006). A adoção desta tecnologia tem possibilitado ao agricultor a simplificação no controle de plantas daninhas.

A soja é cultivada em praticamente todas as regiões do Paraná, as quais apresentam grande diversidade ambiental. Apesar dessa diversidade, a produtividade média obtida nos vários ambientes de teste é o critério de recomendação de cultivares, em programas de melhoramento de soja que buscam selecionar genótipos de adaptação ampla de três grupos de maturação: precoce, até 115 dias da emergência à maturação; semiprecoce, de 116 até 125 dias; e médio, de 126 até 137 dias. Genótipos de adaptação ampla facilitam o processo de transferência de tecnologia e a logística de produção de sementes.

A indicação de uma cultivar, para todas as regiões do Paraná, desconsidera opções de cultivares com adaptações específicas a um tipo de ambiente, como por exemplo, para a região Norte/Oeste, de clima mais quente, ou para a região Sul, de altitudes maiores e clima mais ameno. A possibilidade de estratificação do Estado em sub-regiões com características ambientais semelhantes, para fins de seleção e indicação de genótipos de soja, pode ser verificada a partir de estudos de interações GxA (Allard et al., 1964; Arantes, 1979; Ramalho et al., 1993; Alliprandini et al., 1994; Carvalho et al., 2002; Toledo et al., 2006).

Atualmente, no programa de melhoramento genético da Embrapa Soja, as linhagens convencionais e transgênicas são selecionadas no Paraná em testes de valor de cultivo e uso (VCU), realizados em aproximadamente 15 locais em cada ano, por no mínimo três anos consecutivos. Esse grande número de ambientes de avaliação acarreta custos - financeiro e técnico elevados. Os estudos de interações GxA podem, também, detectar que é possível se reduzir o número de testes de $\mathrm{VCU}$, ao se identificarem locais onde os genótipos apresentam comportamentos similares.

Os objetivos deste trabalho foram: comparar a produtividade e a adaptação de genótipos de soja convencional e de soja transgênica resistente ao herbicida glifosato, de diferentes grupos de maturação, desenvolvidos pelo programa de melhoramento da Embrapa Soja, para o Estado do Paraná; estudar a importância relativa dos efeitos de local, ano, cultivar e suas respectivas interações; e verificar a possibilidade de se estratificar o Estado em regiões mais homogêneas, para reduzir o número de locais nos ensaios de competição de linhagens (testes de VCU).

\section{Material e Métodos}

Dados de produtividade de soja, dos ensaios finais da Embrapa Soja, foram obtidos de 15 locais representativos do Estado do Paraná, nos anos agrícolas de 2001/2002 a 2004/2005 (Tabela 1). Entre os 15 locais, estão os municípios de Mafra no Estado de Santa Catarina e Pedrinhas no Estado de São Paulo, por apresentar características geográficas e climáticas similares aos das regiões Sul e Norte/Oeste do Estado do Paraná, respectivamente.

Tabela 1. Dados dos locais dos ensaios regionais da Embrapa Soja, conduzidos no Estado do Paraná, entre os anos agrícolas 2001/2002 e 2004/2005.

\begin{tabular}{llcl}
\hline Local & Localização & Altitude $(\mathrm{m})$ & Classe de solos \\
\hline Brasilândia & $24^{\circ} 15^{\prime} \mathrm{S} / 53^{\circ} 17^{\prime} \mathrm{W}$ & 485 & Argissolo Vermelho -Amarelo eutrófico \\
Cambará & $23^{\circ} 48^{\prime} \mathrm{S} / 50^{\circ} 02^{\prime} \mathrm{W}$ & 461 & Nitossolo Vermelho eutroférrico \\
Londrina & $23^{\circ} 18^{\prime} \mathrm{S} / 51^{\circ} 25^{\prime} \mathrm{W}$ & 585 & Latossolo Vermelho distroférrico \\
Mandaguaçu & $23^{\circ} 13^{\prime} \mathrm{S} / 51^{\circ} 59^{\prime} \mathrm{W}$ & 427 & Latossolo Vermelho distrófico \\
Palotina & $24^{\circ} 17^{\prime} \mathrm{S} / 53^{\circ} 55^{\prime} \mathrm{W}$ & 370 & Latossolo Vermelho eutroférrico \\
Pedrinhas & $22^{\circ} 48^{\prime} \mathrm{S} / 50^{\circ} 42^{\prime} \mathrm{W}$ & 346 & Nitossolo Vermelho eutroférrico \\
Campo Mourão & $24^{\circ} 03^{\prime} \mathrm{S} / 52^{\circ} 22^{\prime} \mathrm{W}$ & 616 & Latossolo Vermelho distroférrico \\
Cascavel & $24^{\circ} 57^{\prime} \mathrm{S} / 53^{\circ} 28^{\prime} \mathrm{W}$ & 720 & Latossolo Vermelho distroférrico \\
Luiziânia & $24^{\circ} 02^{\prime} \mathrm{S} / 52^{\circ} 22^{\prime} \mathrm{W}$ & 585 & Latossolo Vermelho distroférrico \\
Castro & $24^{\circ} 51^{\prime} \mathrm{S} / 49^{\circ} 56^{\prime} \mathrm{W}$ & 1.053 & Latossolo Vermelho -Amarelo distrófico \\
Guarapuava & $25^{\circ} 23^{\prime} \mathrm{S} / 51^{\circ} 30^{\prime} \mathrm{W}$ & 1.098 & Latossolo Bruno alumínico \\
Mafra & $26^{\circ} 06^{\prime} \mathrm{S} / 49^{\circ} 48^{\prime} \mathrm{W}$ & 792 & Cambissolo Háplico alumínico \\
Mangueirinha & $25^{\circ} 56^{\prime} \mathrm{S} / 52^{\circ} 11^{\prime} \mathrm{W}$ & 940 & Latossolo Vermelho distrófico \\
Ponta Grossa & $25^{\circ} 05^{\prime} \mathrm{S} / 50^{\circ} 01^{\prime} \mathrm{W}$ & 969 & Latossolo Vermelho distrófico \\
Tibagi & $24^{\circ} 32^{\prime} \mathrm{S} / 50^{\circ} 18^{\prime} \mathrm{W}$ & 992 & Latossolo Vermelho distrófico \\
\hline
\end{tabular}


Os materiais utilizados constituíram-se de linhagens e variedades convencionais dos grupos de maturação precoce (até 115 dias), semiprecoce (116 a 125 dias) e médio (126 a 137 dias), e transgênicas semiprecoces e médias no total de 267 . As 23 cultivares comerciais, que foram utilizadas como padrões, são: BRS 232, BRS 230, BRS 215, BRS 184, BRS 183, BRS 153, BRS 134, BRS 133, BRS 247 RR, BRS 246 RR, BRS 245 RR, BRS 244 RR, BRS 243 RR, BRS 242 RR, BRS Valiosa RR, BR-36RCH/S, IAS-5, EMBRAPA 59, EMBRAPA 58, EMBRAPA 48, CD 206, CD 202 e MSOY-7501. As linhagens em teste e os padrões não foram utilizados em todos os anos e locais. A seleção desses materiais como padrões foi baseada no critério de participação no maior número de experimentos, para diminuir o desbalanceamento das análises de variância.

O delineamento experimental utilizado foi o de blocos completos ao acaso, com quatro repetições. As parcelas foram formadas por quatro linhas de $5 \mathrm{~m}$, espaçadas em 0,5 m entre si, com densidade de plantas entre $14 \mathrm{e}$ 18 plantas por metro linear. Quatro metros das duas linhas centrais - foi descartado $0,5 \mathrm{~m}$ em cada extremidade - constituíram a área útil da parcela de $4 \mathrm{~m}^{2}$. As semeaduras de todos os ensaios ocorreram entre 20 de outubro e 15 de novembro, época recomendada para o Estado do Paraná, e os experimentos foram manejados de modo a manter as plantas sob condições normais de desenvolvimento.

Foram realizadas análises de variância individuais para cada ano e local, com os dois tipos (convencional e transgênica) de soja. Após se verificar a homogeneidade de variância, análises conjuntas foram realizadas. Variâncias residuais foram consideradas homogêneas, quando a taxa entre o maior e menor valor foi inferior a 7 (Pimentel-Gomes, 1985). Os efeitos de genótipo, ano e local foram considerados aleatórios, e o efeito de tipo, fixo. As significâncias dos efeitos foram verificadas pelo teste F. As análises estatísticas foram feitas com o procedimento PROC GLM do módulo de estatística do SAS (SAS Institute, 1990).

A possibilidade de redução do número de locais, nos ensaios de competição de linhagens (VCU), foi observada, tendo-se utilizado o método de Lin (1982). A possibilidade de se realizar a estratificação do Estado do Paraná, em regiões mais homogêneas, foi verificada pela significância da interação genótipo $\mathrm{x}$ ambiente, em pares de locais de uma mesma região e de regiões distintas. Essa estratificação foi realizada com três regiões do Estado: Norte/Oeste - Londrina, Cambará, Brasilândia, Mandaguaçu, Palotina e Pedrinhas; Intermediária - Campo Mourão, Cascavel e Luiziânia; e Sul-Castro, Guarapuava, Mafra, Mangueirinha, Ponta Grossa e Tibagi. Foi observado se os locais de uma região apresentavam maior similaridade (interação genótipo $\mathrm{x}$ ambiente não significativa) entre si do que entre locais de regiões distintas. Para a estratificação do Estado em regiões mais homogêneas, o efeito de local foi considerado aleatório. Contudo, para redução de números de locais em testes de VCU, seu efeito foi considerado fixo. As significâncias das interações foram verificadas pelo teste $\mathrm{F}$.

\section{Resultados e Discussão}

A análise de variância conjunta das safras - 2001/ 2002, 2002/2003, 2003/2004 e 2004/2005 - indicou que não houve diferença significativa de produtividade, entre a soja convencional e a transgênica, para os grupos de maturação semiprecoce e médio (Tabela 2). Embora o programa de desenvolvimento da soja RR seja recente, em comparação ao da soja convencional, a similaridade entre as médias ocorreu provavelmente em razão do método de melhoramento adotado pela Embrapa Soja, que introduziu o gene de resistência ao herbicida (RR), por retrocruzamento, nas melhores cultivares comerciais. Não houve, também, diferença significativa entre os três

Tabela 2. Análise de variância de produtividade de diferentes tipos de soja (convencional e transgênica), de ciclos semiprecoce e médio, no Estado do Paraná, entre os anos agrícolas 2001/2002 e 2004/2005.

\begin{tabular}{|c|c|c|c|c|}
\hline \multirow[t]{2}{*}{ Fonte de variação } & \multicolumn{2}{|c|}{ Semiprecoce } & \multicolumn{2}{|r|}{ Médio } \\
\hline & GL & QM & GL & QM \\
\hline Tipo (T) & 1 & $123.057 .163^{\mathrm{ns}}$ & 1 & $21.799 .941^{\mathrm{n}}$ \\
\hline Ano (A) & 3 & $484.307 .357^{\mathrm{ns}}$ & 3 & $216.617 .528^{n}$ \\
\hline $\mathrm{TxA}$ & 3 & $30.471 .231^{* *}$ & 3 & $16.415 .222^{n}$ \\
\hline Local (L) & 14 & $297.391 .827^{*}$ & 14 & $86.965 .865^{\mathrm{n}}$ \\
\hline TxL & 14 & $7.953 .406^{\mathrm{ns}}$ & 14 & $9.120 .688^{\mathrm{n}}$ \\
\hline AxL & 33 & $136.882 .591^{* *}$ & 25 & $82.305 .351^{* *}$ \\
\hline TxAxL & 33 & $5.959 .918^{* *}$ & 25 & $8.125 .383^{*}$ \\
\hline Genótipo - G (tipo) & 201 & $1.502 .021^{* *}$ & 103 & $1.513 .156^{*}$ \\
\hline $\mathrm{AxG}$ (tipo) & 107 & $521.534^{\mathrm{ns}}$ & 47 & 574.521 * \\
\hline LxG (tipo) & 2.001 & $587.379^{* *}$ & 996 & $526.749^{*}$ \\
\hline AxLxG (tipo) & 1.020 & $478.224^{* *}$ & 349 & $357.856^{* *}$ \\
\hline Erro & 9.457 & 197.950 & 4.407 & 211.155 \\
\hline$\overline{C V(\%)}$ & & 14,79 & & 15,40 \\
\hline Média & & 3.007 & & 2.983 \\
\hline
\end{tabular}

${ }^{n}$ Não-significativo. $*$ e $* *$ Significativo a 5 e $1 \%$ de probabilidade, respectivamente, pelo teste $\mathrm{F}$. 
grupos de maturação - precoce $\left(3.172 \mathrm{~kg} \mathrm{ha}^{-1}\right)$, semiprecoce (3.177 kg ha-1) e médio $\left(3.048 \mathrm{~kg} \mathrm{ha}^{-1}\right)$ da soja convencional, e entre os dois grupos de maturação - semiprecoce $\left(2.945 \mathrm{~kg} \mathrm{ha}^{-1}\right)$ e médio $\left(2.924 \mathrm{~kg} \mathrm{ha}^{-1}\right)$ - da soja transgênica (Tabelas 3 e 4). A similaridade de produtividade entre linhagens convencionais e transgênicas, e entre as linhagens nos grupos de maturação, facilita a seleção do tipo e do grupo de maturação da soja por parte dos agricultores, fato que permite variações de herbicida e escalonamento da semeadura.

Para os dois tipos de soja, o efeito de ano foi não significativo para as linhagens dos grupos de maturação semiprecoce e médio, e o efeito de local foi significativo, a 5\% de probabilidade, apenas para o grupo semiprecoce (Tabela 2). Os efeitos da interação ano por local foram significativos a $1 \%$ de probabilidade, para os dois grupos de maturação, o que indica, a exemplo de análises anteriores (Alliprandini et al., 1994), que os efeitos das combinações entre ano e local são importantes para o desempenho produtivo da soja. Considerando-se que cada local e ano caracterizam um ambiente diferente, pode-se assumir que a produtividade das linhagens foi afetada pelas mudanças do ambiente. A interação tripla tipo por ano por local foi significativa para os dois grupos de maturação (semiprecoce e médio), o que indica que as linhagens convencionais e transgênicas responderam de forma diferenciada às variações do ambiente. A interação ano por genótipo, dentro de tipo, não foi significativa para os genótipos semiprecoces, e foi significativa a $1 \%$ para os do grupo de maturação médio, o que indica maior susceptibilidade dos genótipos do grupo médio às variações de ano. Esses dados estão de acordo com observações anteriores, obtidas dentro do programa de melhoramento de soja da Embrapa Soja, que mostram que os materiais genéticos de ciclo semiprecoce são melhor adaptados aos ambientes do Estado do Paraná e, portanto, apresentam interações menores com os efeitos de ano e local. Os efeitos da interação entre local e genótipo, dentro de tipo, e da interação entre ano, local e genótipo, dentro de tipo, foram significativos a $1 \%$ de probabilidade, para os dois grupos de maturação. Os resultados das interações acima indicam que: a combinação entre anos e locais foi importante para o desempenho dos genótipos; e que os efeitos de local são relativamente mais importantes que os de ano. Essa mudança de desempenho produtivo das linhagens de soja, nos diferentes ambientes avaliados, mostra haver possibilidade de ganho em produtividade, se for realizada a estratificação ambiental (Carvalho et al., 2002).

Considerando-se os tipos de linhagem separadamente, as análises conjuntas de anos e locais para os ensaios de soja convencional (Tabela 3) e transgênica (Tabela 4) indicaram que o efeito de ano não foi significativo para os grupos de maturação, e que o efeito de local foi significativo apenas para os grupos de maturação precoce e semiprecoce da soja convencional. Uma possível explicação para essa aparente maior estabilidade da soja transgênica foi o fato de as linhagens transgênicas testadas serem essencialmente derivadas, por retrocruzamentos, de cultivares produtivas e estáveis. As interações de anos com genótipos não foram significativas quanto aos grupos de maturação da soja convencional e transgênica, exceto para o grupo médio da soja convencional. As interações entre local e

Tabela 3. Análise de variância de produtividade de soja convencional de ciclos precoce, semiprecoce e médio, no Estado do Paraná, entre os anos agrícolas 2001/2002 e 2004/2005.

\begin{tabular}{|c|c|c|c|c|c|c|}
\hline \multirow[t]{2}{*}{ Fonte de variação } & \multicolumn{2}{|c|}{ Precoce } & \multicolumn{2}{|c|}{ Semiprecoce } & \multicolumn{2}{|c|}{ Médio } \\
\hline & GL & QM & GL & QM & GL & QM \\
\hline$\overline{\text { Ano (A) }}$ & 3 & $51.472 .350^{\mathrm{ns}}$ & 3 & $114.374 .947^{\mathrm{ns}}$ & 3 & $81.476 .989^{\text {ns }}$ \\
\hline Local (L) & 14 & $115.337 .102^{* *}$ & 14 & $77.861 .835^{*}$ & 14 & $42.632 .243^{\mathrm{ns}}$ \\
\hline $\mathrm{AxL}$ & 32 & $32.773 .135^{* *}$ & 33 & $40.433 .598^{* *}$ & 25 & $46.374 .559^{* *}$ \\
\hline Bloco/A/L & 150 & $733.961^{* *}$ & 153 & $625.116^{* *}$ & 129 & $656.001^{* *}$ \\
\hline Genótipos (G) & 51 & $1.362 .910^{* *}$ & 42 & $2.685 .855^{* *}$ & 43 & $1.785 .237^{\mathrm{ns}}$ \\
\hline $\mathrm{AxG}$ & 13 & $267.751^{\mathrm{ns}}$ & 20 & $433.363^{\mathrm{ns}}$ & 19 & $886.040^{* *}$ \\
\hline $\mathrm{LxG}$ & 630 & $376.286^{* *}$ & 504 & $608.713^{* *}$ & 464 & $579.042^{* *}$ \\
\hline AxLxG & 120 & $254.599^{* *}$ & 210 & $321.065^{* *}$ & 156 & $362.364^{* *}$ \\
\hline$\overline{\text { Erro }}$ & 2.341 & 209.367 & 2.221 & 198.131 & 1.936 & 240.186 \\
\hline$\overline{\mathrm{CV}(\%)}$ & & 13,06 & & 14,01 & & 15,08 \\
\hline Média & & $3.172^{\mathrm{ns}}$ & & $3.177^{\mathrm{ns}}$ & & $3.048^{\mathrm{ns}}$ \\
\hline
\end{tabular}

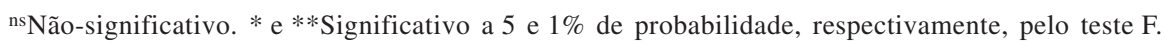


genótipo foram significativas a 1 e $5 \%$ de probabilidade, respectivamente, quanto aos tipos de soja convencional e transgênico, em todos os grupos de maturação. Esses resultados indicaram que os efeitos de local, provavelmente, afetaram mais o comportamento das linhagens que os efeitos de ano, o que contrasta com Alliprandini et al. (1994), que relataram que anos e locais tinham influência significativa e intercambiáveis sobre o desempenho dos genótipos.

A interação tripla foi significativa a $1 \%$ de probabilidade, para os dois tipos de soja, em todos os grupos de maturação (Tabelas 3 e 4). Esses resultados confirmaram as informações da análise conjunta da Tabela 2, e indicam que as combinações de anos e locais formaram ambientes que influenciaram diferencialmente o comportamento das linhagens e que, na composição desses ambientes, o efeito de local é mais importante que o de anos. Portanto, o melhorista deve preferir aumentar o número de locais de teste do que anos em seu trabalho de avaliação de linhagens. Os resultados indicam, também, que uma divisão do Estado do Paraná, em regiões homogêneas, poderia ser útil aos trabalhos de avaliação, para a indicação de cultivares específicas para cada região (Alliprandini et al., 1994; Carvalho et al., 2002; Murakami \& Cruz, 2004).

Com base na significância da interação GxA, para cada par de locais de teste das linhagens convencionais do grupo semiprecoce, observou-se que os locais da região Norte/Oeste apresentaram menor similaridade entre si, em relação à similaridade apresentada pelos locais das regiões Intermediária e Sul (Tabela 5).

Tabela 4. Análise de variância de produtividade de soja transgênica de ciclos semiprecoce e médio, no Estado do Paraná, entre os anos agrícolas 2001/2002 e 2004/2005.

\begin{tabular}{|c|c|c|c|c|}
\hline \multirow[t]{2}{*}{ Fonte de variação } & \multicolumn{2}{|c|}{ Semiprecoce } & \multicolumn{2}{|r|}{ Médio } \\
\hline & GL & QM & GL & QM \\
\hline Ano (A) & 3 & $217.726 .575^{\mathrm{ns}}$ & 3 & $71.636 .856^{\mathrm{ns}}$ \\
\hline Local (L) & 14 & $126.307 .591^{\mathrm{ns}}$ & 14 & $32.711 .204^{\mathrm{ns}}$ \\
\hline $\mathrm{AxL}$ & 33 & $87.950 .623^{* *}$ & 25 & $39.822 .345^{* *}$ \\
\hline Bloco/A/L & 154 & $661.540^{* *}$ & 129 & $477.031^{* *}$ \\
\hline Genótipos (G) & 87 & $1.460 .391^{* *}$ & 36 & $1.378 .252^{* *}$ \\
\hline $\mathrm{AxG}$ & 61 & $634.643^{\mathrm{ns}}$ & 17 & $455.452^{\mathrm{ns}}$ \\
\hline LxG & 1.109 & $584.463^{*}$ & 412 & $452.539^{*}$ \\
\hline AxLxG & 668 & $501.164^{* *}$ & 145 & $344.764^{* *}$ \\
\hline Erro & 5.563 & 174.645 & 1.748 & 168.551 \\
\hline CV (\%) & & 14,19 & & 14,04 \\
\hline Média & & $2.945^{\mathrm{ns}}$ & & $2.924^{\mathrm{ns}}$ \\
\hline
\end{tabular}

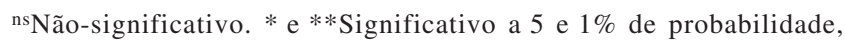
respectivamente, pelo teste $\mathrm{F}$.
Na região Norte/Oeste, a freqüente ocorrência de interação GxA significativa refletiu o comportamento distinto dos genótipos nos diferentes locais de teste. Esses resultados demonstram a grande diversidade ambiental (altitude, solo, temperatura, precipitação pluvial) dessa região e indicam haver a necessidade de estabelecimento de um número maior de locais de teste. A interação entre locais dessa região, com os locais das demais regiões, tendeu, também, a ser significativa. Entretanto, um número maior de pares de locais com interação GxA não-significativa, na região Sul, refletiu a maior homogeneidade de ambientes e a possibilidade de se reduzir o número de locais de teste. A similaridade entre locais da região Sul foi maior que a desses locais com os de outras regiões, o que mostra ser interessante, do ponto de vista dos testes de VCU, se realizar uma regionalização no Estado. Nessa região, o local que mostrou maior dissimilaridade dos demais pontos de teste foi Tibagi, que apresentou condições ambientais distintas. Na região Intermediária, a análise foi prejudicada, em razão do número reduzido de locais de teste. Contudo, houve similaridade entre Campo Mourão e Luiziânia, e menor similaridade desses locais com Cascavel. Luiziânia apresentou maior similaridade com os locais da região Sul, em comparação aos da região Norte/Oeste. Resultados similares ao do grupo de maturação semiprecoce foram obtidos para os demais grupos de maturação.

Ao se analisar o comportamento das linhagens semiprecoces transgênicas, observou-se maior similaridade entre os locais de teste, em comparação com as análises das linhagens convencionais (Tabelas 5 e 6). Uma possível explicação para esse resultado é que, conforme mencionado anteriormente, as linhagens transgênicas testadas são essencialmente derivadas de cultivares produtivas e estáveis, que foram cuidadosamente selecionadas para entrar no processo de incorporação do gene RR por programa de retrocruzamentos. Mesmo com razoável similaridade entre os locais testados, verificou-se que os locais da região Sul, exceto Tibagi, foram um pouco mais similares entre si, em comparação com a similaridade obtida para os locais da região Norte/Oeste. Contudo, a estratificação do Estado do Paraná, para avaliar linhagens transgênicas semiprecoces de soja, foi menos evidente que a observada para linhagens convencionais. De outro modo, o número de locais de uma mesma região, com interação genótipo $\mathrm{x}$ ambiente não-significativa, não 
foi tão superior à observada entre locais de regiões distintas. Isto foi verificado, também, para os outros grupos de maturação.

Quanto à possibilidade de descarte de locais de teste, Ponta Grossa e Guarapuava, na região Sul, e Campo Mourão e Luiziânia, na região Intermediária, tiveram interação genótipo $\mathrm{x}$ ambiente nãosignificativa, nos quatro anos de teste, na análise das linhagens convencionais semiprecoces (Tabelas 5 e 7). Essa similaridade indica que há possibilidade de descarte de um local de cada região, sem muito prejuízo na comparação das linhagens. No entanto, como o fenômeno não se repetiu na análise dos transgênicos (Tabelas 6 e 8), não há indicação de repetibilidade de resultados dessa eliminação. Resultados similares foram obtidos para os outros grupos de maturação.
Ao formar grupos de locais com interação nãosignificativa, com base na metodologia de Lin (1982), pode-se agrupar um número maior de locais, nas análises de linhagens transgênicas, do que nas das convencionais (Tabelas 7 e 8). Observou-se, também, que em anos chuvosos (por exemplo, 2002/2003), houve maior agrupamento de locais das três regiões, independentemente do tipo de linhagem (convencional e transgênica). No entanto, em anos menos chuvosos, houve tendência de estratificação do Estado, com maior similaridade apenas entre os locais da região Sul, nas análises de linhagens convencionais. Esse fato ocorreu para todos os grupos de maturação e corrobora observação de Alliprandini et al. (1994), que citaram que o balanço de água no solo tem importância fundamental para o comportamento das linhagens, nos vários locais.

Tabela 5. Interação genótipo x ambiente de locais avaliados nos ensaios regionais de genótipos de soja convencional, do grupo de maturação semiprecoce, da Embrapa Soja, conduzidos no Estado do Paraná entre os anos agrícolas 2001/2002 e 2004/2005.

\begin{tabular}{|c|c|c|c|c|c|c|c|c|c|c|c|c|c|c|}
\hline Local & Cambará & $\begin{array}{l}\text { Londrina } \\
\end{array}$ & Mandaguaçu & Palotina & Pedrinhas & C. Mourão & $\begin{array}{l}\text { Cascavel } \\
\end{array}$ & Luiziana & Castro & Guarapuava & Mafra & Mangueirinha & P. Grossa & Tibagi \\
\hline \multirow[t]{2}{*}{ Brasilândia } & $2004 *$ & $2004 *$ & $2004^{\text {ns }}$ & 2004* & $-^{(1)}$ & $2004 *$ & $2004 *$ & $2004^{\text {ns }}$ & 2004* & $2004^{\text {ns }}$ & $2004^{15}$ & - & $2004^{\text {ns }}$ & - \\
\hline & - & $2005^{*}$ & - & $2005^{*}$ & - & $2005^{*}$ & $2005^{*}$ & - & - & $2005^{*}$ & $2005 *$ & $2005^{*}$ & $2005^{*}$ & - \\
\hline \multirow[t]{3}{*}{ Cambará } & & 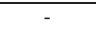 & $2002 *$ & $2002^{*}$ & $2002^{*}$ & & $2002^{*}$ & $2002^{*}$ & $2002^{*}$ & $2002^{*}$ & $2002 *$ & $2002^{*}$ & $2002^{*}$ & - \\
\hline & & $2003^{\text {ns }}$ & $2003 *$ & $2003^{\text {ns }}$ & $2003^{*}$ & $2003^{*}$ & $2003^{*}$ & $2003 *$ & $2003^{\text {ns }}$ & $2003 *$ & $2003^{\text {ns }}$ & $2003^{*}$ & $2003^{\text {ns }}$ & 2003 \\
\hline & & $2004 *$ & $2004 *$ & $2004 *$ & - & 2004* & $2004 *$ & $2004 *$ & $2004 *$ & $2004 *$ & $2004 *$ & - & $2004 *$ & - \\
\hline \multirow[t]{3}{*}{ Londrina } & & & $2003^{\text {ns }}$ & $2003^{\text {ns }}$ & $2003^{*}$ & $2003^{*}$ & $2003^{*}$ & $2003^{\text {ns }}$ & $2003 *$ & $2003^{\text {ns }}$ & $2003^{\text {15 }}$ & $2003^{\text {ns }}$ & $2003^{\text {ns }}$ & $2003^{*}$ \\
\hline & & & $2004 *$ & $2004 *$ & - & $2004 *$ & $2004 *$ & $2004 *$ & 2004* & 2004* & $2004 *$ & - & $2004 *$ & - \\
\hline & & & - & $2005^{*}$ & - & $2005^{*}$ & $2005^{\mathrm{ns}}$ & - & - & $2005^{*}$ & $2005 *$ & $2005 *$ & $2005^{*}$ & - \\
\hline \multirow[t]{3}{*}{ Mandaguaçu } & & & & $2002^{*}$ & $2002^{*}$ & - & $2002^{*}$ & $2002^{15}$ & $2002^{\text {ns }}$ & $2002^{\text {ns }}$ & $2002 *$ & $2002^{*}$ & $2002^{\text {ns }}$ & - \\
\hline & & & & $2003^{*}$ & 2003* & 2003* & $2003^{\text {ns }}$ & $2003^{\text {15 }}$ & 2003* & 2003* & $2003^{\text {ns }}$ & $2003 *$ & 2003* & $2003^{*}$ \\
\hline & & & & $2004^{\text {ns }}$ & - & $2004^{\text {ns }}$ & $2004 *$ & $2004^{\text {ns }}$ & $2004 *$ & $2004 *$ & $2004 *$ & - & 2004* & - \\
\hline \multirow[t]{4}{*}{ Palotina } & & & & & $2002^{*}$ & - & $2002^{*}$ & $2002^{*}$ & $2002 *$ & $2002^{\mathrm{ns}}$ & $2002^{\text {ns }}$ & $2002 *$ & $2002^{*}$ & - \\
\hline & & & & & $2003^{*}$ & $2003^{*}$ & 2003* & $2003^{\text {15 }}$ & 2003* & $2003 *$ & $2003^{\text {ns }}$ & $2003 *$ & $2003 *$ & $2003^{*}$ \\
\hline & & & & & - & $2004^{\text {ns }}$ & $2004^{\mathrm{ns}}$ & $2004^{\text {15 }}$ & 2004* & 2004* & $2004 *$ & - & $2004 *$ & - \\
\hline & & & & & - & $2005^{*}$ & $2005^{*}$ & - & - & $2005^{*}$ & $2005^{*}$ & $2005 *$ & $2005^{*}$ & - \\
\hline \multirow{2}{*}{ Pedrinhas } & & & & & & & $2002^{*}$ & $2002^{*}$ & $2002^{*}$ & $2002^{*}$ & $2002^{*}$ & $2002^{*}$ & $2002^{*}$ & - \\
\hline & & & & & & $2003^{*}$ & $2003^{*}$ & $2003^{*}$ & $2003 *$ & $2003 *$ & $2003^{\text {ns }}$ & $2003 *$ & $2003^{*}$ & $2003^{*}$ \\
\hline \multirow[t]{3}{*}{ Campo Mourão } & & & & & & & $2003^{*}$ & $2003^{15}$ & $2003^{\text {ns }}$ & $2003 *$ & $2003^{\text {ns }}$ & $2003^{*}$ & $2003^{*}$ & $2003^{*}$ \\
\hline & & & & & & & $2004^{\text {ns }}$ & $2004^{\text {15 }}$ & 2004* & 2004* & $2004 *$ & - & $2004 *$ & - \\
\hline & & & & & & & $2005^{*}$ & - & - & $2005^{\mathrm{ns}}$ & $2005^{*}$ & $2005^{\text {ns }}$ & $2005^{*}$ & - \\
\hline \multirow[t]{4}{*}{$\begin{array}{l}\text { Cascavel } \\
\text { n }\end{array}$} & & & & & & & & $2002^{*}$ & $2002 *$ & $2002 *$ & $2002 *$ & $2002 *$ & $2002 *$ & - \\
\hline & & & & & & & & $2003^{*}$ & $2003 *$ & $2003 *$ & $2003 *$ & $2003 *$ & $2003^{*}$ & $2003^{*}$ \\
\hline & & & & & & & & $2004^{\text {ns }}$ & 2004* & 2004* & $2004 *$ & - & $2004 *$ & - \\
\hline & & & & & & & & - & - & $2005^{*}$ & $2005^{*}$ & $2005^{\mathrm{ns}}$ & $2005^{\mathrm{ns}}$ & - \\
\hline \multirow[t]{3}{*}{ Luiziana } & & & & & & & & & $2002^{\text {ns }}$ & $2002^{\mathrm{ns}}$ & $2002^{\text {ns }}$ & $2002^{*}$ & $2002^{\text {ns }}$ & - \\
\hline & & & & & & & & & 2003* & $2003^{\text {ns }}$ & $2003^{\text {ns }}$ & $2003^{\text {ns }}$ & $2003^{\text {ns }}$ & $2003^{\text {ns }}$ \\
\hline & & & & & & & & & $2004 *$ & $2004^{\mathrm{ns}}$ & $2004^{\text {ns }}$ & - & $2004^{\text {ns }}$ & - \\
\hline \multirow[t]{3}{*}{$\begin{array}{l}\text { Castro } \\
\end{array}$} & & & & & & & & & & $2002^{\text {ns }}$ & $2002 *$ & $2002^{*}$ & $2002^{\text {ns }}$ & - \\
\hline & & & & & & & & & & $2003 *$ & $2003^{\text {ns }}$ & 2003* & $2003^{\text {ns }}$ & $2003^{*}$ \\
\hline & & & & & & & & & & $2004 *$ & 2004* & - & $2004 *$ & - \\
\hline \multirow[t]{4}{*}{ Guarapuava } & & & & & & & & & & & $2002^{\text {ns }}$ & $2002^{*}$ & $2002^{\text {ns }}$ & - \\
\hline & & & & & & & & & & & $2003^{\text {ns }}$ & $2003^{\text {ns }}$ & $2003^{\text {ns }}$ & $2003^{*}$ \\
\hline & & & & & & & & & & & $2004^{\text {ns }}$ & - & $2004^{\text {ns }}$ & - \\
\hline & & & & & & & & & & & $2005^{*}$ & $2005^{\mathrm{ns}}$ & $2005^{*}$ & - \\
\hline \multirow[t]{4}{*}{$\overline{\text { Mafra }}$} & & & & & & & & & & & & $2002^{*}$ & $2002^{\text {ns }}$ & - \\
\hline & & & & & & & & & & & & $2003^{\text {ns }}$ & $2003^{\text {ns }}$ & $2003^{\text {ns }}$ \\
\hline & & & & & & & & & & & & - & $2004^{\text {ns }}$ & - \\
\hline & & & & & & & & & & & & $2005^{*}$ & $2005^{*}$ & - \\
\hline \multirow[t]{3}{*}{ Mangueirinha } & & & & & & & & & & & & & $2002^{*}$ & - \\
\hline & & & & & & & & & & & & & $2003^{\text {ns }}$ & $2003^{*}$ \\
\hline & & & & & & & & & & & & & $2005^{\text {ns }}$ & - \\
\hline Ponta Grossa & & & & & & & & & & & & & & $2003^{*}$ \\
\hline
\end{tabular}

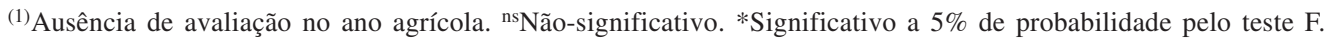


Tabela 6. Interação genótipo x ambiente de locais, avaliados nos ensaios regionais de genótipos de soja convencional, do grupo de maturação semiprecoce, da Embrapa Soja, conduzidos no Estado do Paraná entre os anos agrícolas 2001/2002 e 2004/2005.

\begin{tabular}{|c|c|c|c|c|c|c|c|c|c|c|c|c|c|c|}
\hline Local & Cambará & Londrina & Mandaguaçu & Palotina & Pedrinhas & C. Mourão & Cascavel & Luiziana & Castro & Guarapuava & Mafra & Mangueirinha & P. Grossa & Tibagi \\
\hline \multirow[t]{3}{*}{ Brasilândia } & RR2003* & RR2003* & RR2003* & RR2003 $3^{\text {ns }}$ & RR2003 $3^{\text {ns }}$ & $\mathrm{RR}^{2003^{\text {ns }}}$ & RR2003* & RR2003* & RR2003* & RR2003* & RR2003* & RR2003* & RR2003* & RR2003* \\
\hline & RR2004* & RR2004* & RR2004 $4^{\text {ns }}$ & RR2004* & $-^{(1)}$ & RR2004 $4^{\text {ns }}$ & RR2004 $4^{\text {ns }}$ & RR2004* & RR2004* & RR2004* & RR2004* & RR2004* & RR2004* & - \\
\hline & - & RR2005* & - & RR2005* & - & RR2005* & RR2005* & - & - & RR2005* & RR2005* & RR2005* & RR2005* & - \\
\hline \multirow[t]{3}{*}{ Cambará } & & RR2002 $2^{\text {ns }}$ & RR2002* & RR2002 $2^{\text {ns }}$ & RR2002* & RR2002 $2^{\text {ns }}$ & RR2002 $2^{\text {ns }}$ & RR2002 ${ }^{\text {ns }}$ & RR2002 $2^{\text {ns }}$ & RR2002 $2^{\text {ns }}$ & RR2002* & RR2002* & RR2002 $2^{\text {ns }}$ & RR2002* \\
\hline & & RR2003* & RR2003* & RR2003 ${ }^{\text {ns }}$ & RR2003 ${ }^{\text {ns }}$ & RR2 $2003^{\text {ns }}$ & RR2003 ${ }^{\text {ns }}$ & RR $2003^{\text {ns }}$ & RR $2003^{\text {ns }}$ & RR2003 $3^{\text {ns }}$ & RR2003 ${ }^{\text {ns }}$ & RR2003 $3^{\text {ns }}$ & RR2003 ${ }^{\text {ns }}$ & RR2003* \\
\hline & & RR2004* & RR2004 $4^{\text {ts }}$ & RR2004 $4^{\text {ns }}$ & - & RR2004* & RR2004* & RR2004 & RR2004* & RR2004 $4^{\text {ns }}$ & RR2004 $4^{\text {ns }}$ & RR2004 $4^{\text {ns }}$ & RR2004 $4^{\text {ns }}$ & - \\
\hline \multirow[t]{4}{*}{ Londrina } & & & RR2002 $2^{\text {ns }}$ & RR2002 $2^{\text {ns }}$ & RR2002* & RR2002 $2^{\text {ns }}$ & RR2002* & RR2002* & RR2002 $2^{\text {ns }}$ & RR2002* & RR2002* & RR2002* & RR2002 $2^{\text {ns }}$ & RR2002* \\
\hline & & & RR2003* & RR2003* & RR2003 $3^{\text {ns }}$ & RR2003* & RR2003* & RR2003* & RR2003* & RR2003* & RR2003* & RR2003* & RR2003* & RR2003* \\
\hline & & & RR2004* & RR2004* & - & RR2004* & RR2004* & RR2004* & RR2004* & RR2004* & RR2004* & RR2004 $4^{\text {ns }}$ & RR2004* & - \\
\hline & & & - & RR2005* & - & RR2005* & RR2005* & - & - & RR2 $2005^{\text {ns }}$ & RR2005* & RR2 $2005^{\text {ns }}$ & RR2005* & - \\
\hline \multirow{2}{*}{ Mandaguaçu } & & & & RR2003* & RR2003 ${ }^{\text {ns }}$ & RR2003* & RR2003 ${ }^{\text {ns }}$ & RR2003* & RR2003* & RR2003* & RR2003 $3^{\text {ns }}$ & RR2003* & RR2003 ${ }^{\text {ns }}$ & RR2003* \\
\hline & & & & RR2004 ${ }^{\text {ns }}$ & - & RR2004 ${ }^{\text {ns }}$ & RR2004 ${ }^{\text {ns }}$ & RR2004 & RR $2004^{\text {ns }}$ & RR2004 ${ }^{\text {ns }}$ & RR2004 ${ }^{\text {ns }}$ & RR2004 ${ }^{\text {ns }}$ & RR2004 ${ }^{\text {ns }}$ & - \\
\hline \multirow[t]{4}{*}{ Palotina } & & & & & RR2002* & RR2002* & RR2002 ${ }^{\text {ns }}$ & RR2002 ${ }^{\text {ns }}$ & RR2002 $2^{\text {ns }}$ & RR2002* & RR2002* & RR2002* & RR2002* & RR2002* \\
\hline & & & & & RR2003 $3^{\text {ns }}$ & RR2003 $3^{\text {ns }}$ & RR2003 ${ }^{\text {ns }}$ & RR $2003^{\text {ns }}$ & RR $2003^{\text {ns }}$ & RR2003 $3^{\text {ns }}$ & RR2003 $3^{\text {ns }}$ & RR2 $2003^{\text {ns }}$ & RR2003 ${ }^{\text {ns }}$ & RR2003 \\
\hline & & & & & - & RR2004* & RR2004* & RR2004 ${ }^{\text {ns }}$ & RR2 $2004^{\text {ns }}$ & RR2004 ${ }^{\text {ns }}$ & RR2004 ${ }^{\text {ns }}$ & RR2004 $4^{\text {ns }}$ & RR2004 ${ }^{\text {ns }}$ & - \\
\hline & & & & & - & RR2005* & RR2005* & - & - & RR2005* & RR2005* & RR2005* & RR2005* & - \\
\hline \multirow[t]{2}{*}{ Pedrinhas } & & & & & & RR2002* & RR2002* & RR2002* & RR2002* & RR2002* & RR2002* & RR2002* & RR2002* & RR2002* \\
\hline & & & & & & RR2003 ${ }^{\text {ns }}$ & RR2003 ${ }^{\text {ns }}$ & RR $2003^{\text {ns }}$ & RR $2003^{\text {ns }}$ & RR2 $2003^{\text {ns }}$ & RR2003 $3^{\text {ns }}$ & RR2003* & RR2003* & RR2003* \\
\hline \multirow{3}{*}{\multicolumn{2}{|c|}{ Campo Mourão }} & & & & & & RR2002 ${ }^{\text {ns }}$ & RR2002 ${ }^{\text {ns }}$ & RR2002* & RR2002 ${ }^{\text {ns }}$ & RR2002 $2^{\text {ns }}$ & RR2002* & RR2002* & RR2002* \\
\hline & & & & & & & RR2003 ${ }^{\text {ns }}$ & RR2003 ${ }^{\text {ns }}$ & RR2 $2003^{\text {ns }}$ & RR2003 $3^{\text {ns }}$ & RR2003 $3^{\text {ns }}$ & RR2003* & RR2003* & RR2003* \\
\hline & & & & & & & RR2004 ${ }^{\text {ns }}$ & RR2004* & RR $2004^{\text {ns }}$ & RR2004 ${ }^{\text {ns }}$ & RR2004* & RR2004* & RR2004* & - \\
\hline \multirow{3}{*}{ Cascavel } & & & & & & & & RR $2003^{\text {ns }}$ & RR $2003^{\text {ns }}$ & RR2 $2003^{\text {ns }}$ & RR2003 $3^{\text {ns }}$ & RR2003 $3^{\text {ns }}$ & RR2003 ${ }^{\text {ns }}$ & RR2003* \\
\hline & & & & & & & & RR2004* & RR2004* & RR2004 ${ }^{\text {ns }}$ & RR2004* & RR2004* & RR2004* & - \\
\hline & & & & & & & & - & - & RR2005* & RR2 $005^{\text {ns }}$ & RR2005 $5^{\text {ns }}$ & RR2005 ${ }^{\mathrm{ns}}$ & - \\
\hline \multirow[t]{3}{*}{ Luiziana } & & & & & & & & & RR2002* & RR2002 $2^{\text {ns }}$ & RR2002* & RR2002* & RR2002* & RR2002* \\
\hline & & & & & & & & & RR2 $2003^{\text {ns }}$ & RR2003 ${ }^{\text {ns }}$ & RR2003 $3^{\text {ns }}$ & RR2003* & RR2003* & RR2003* \\
\hline & & & & & & & & & RR2004* & RR2004 ${ }^{\text {ns }}$ & RR2004 $4^{\text {ns }}$ & RR2004 ${ }^{\text {ns }}$ & RR2004 ${ }^{\text {ns }}$ & - \\
\hline \multirow[t]{3}{*}{ Castro } & & & & & & & & & & RR2002* & RR2002* & RR2002* & RR2002* & RR2002* \\
\hline & & & & & & & & & & RR2003 $3^{\text {ns }}$ & RR2003 $3^{\text {ns }}$ & RR2003* & RR2003* & RR2003* \\
\hline & & & & & & & & & & RR2004 ${ }^{\text {ns }}$ & RR2004* & RR2004 $4^{\text {ns }}$ & RR2004* & - \\
\hline \multirow[t]{4}{*}{ Guarapuava } & & & & & & & & & & & RR2002 $2^{\text {ns }}$ & RR2002* & RR2002 ${ }^{\text {ns }}$ & RR2002* \\
\hline & & & & & & & & & & & RR2003 $3^{\text {ns }}$ & RR2003* & RR2003* & RR2003* \\
\hline & & & & & & & & & & & RR2004 ${ }^{\text {ns }}$ & RR2004 ${ }^{\text {ns }}$ & RR2004 ${ }^{\text {ns }}$ & - \\
\hline & & & & & & & & & & & RR2005* & RR2005* & RR2005* & - \\
\hline \multirow[t]{4}{*}{ Mafra } & & & & & & & & & & & & RR2002* & RR2002* & RR2002* \\
\hline & & & & & & & & & & & & RR2003* & RR2003ns & RR2003* \\
\hline & & & & & & & & & & & & RR2004 ${ }^{\text {ns }}$ & RR2004 ${ }^{\text {ns }}$ & - \\
\hline & & & & & & & & & & & & RR2005* & RR2005* & - \\
\hline \multirow{4}{*}{\multicolumn{2}{|c|}{ Mangueirinha }} & & & & & & & & & & & & RR2002* & RR2002* \\
\hline & & & & & & & & & & & & & RR2003* & RR2003* \\
\hline & & & & & & & & & & & & & RR2004 $4^{\text {ns }}$ & - \\
\hline & & & & & & & & & & & & & RR2005 ${ }^{\text {ns }}$ & - \\
\hline \multicolumn{2}{|l|}{ Ponta Grossa } & & & & & & & & & & & & & $\begin{array}{l}\text { RR2002* } \\
\text { RR2003* }\end{array}$ \\
\hline
\end{tabular}

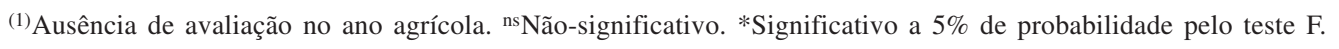

Tabela 7. Estratificação ambiental, com base na discriminação fenotípica relacionada à produtividade de grãos de linhagens de soja convencional, do grupo de maturação semiprecoce, no período de 2001/2002 a 2004/2005, em diversos locais do Paraná(1).

\begin{tabular}{ll}
\hline Ano de avaliação & \multicolumn{1}{c}{ Conjunto de ambientes } \\
\hline $2001 / 2002$ & (Castro, Guarapuava, Ponta Grossa, Luiziânia, Mandaguaçu), (Guarapuava, Mafra, Palotina), (Mafra, Ponta Grossa), \\
& (Luiziânia, Mafra), (Cambará), (Cascavel), (Mangueirinha), (Pedrinhas). \\
\hline 2002/2003 & (Mafra, Ponta Grossa, Luiziânia, Londrina, Mangueirinha, Guarapuava), (Cascavel, Mandaguaçu), (Cambará, Palotina, \\
& Mafra), (Castro, Ponta Grossa, Cambará), (Castro, Mafra, Campo Mourão), (Mafra, Pedrinhas), (Luiziânia, \\
& Mandaguaç), (Cambará, Londrina), (Luiziânia, Palotina), (Londrina, Mandaguaçu), (Campo Mourão, Luiziânia), \\
& (Londrina, Palotina), (Mafra, Mandaguaçu), (Tibagi). \\
\hline 2003/2004 & (Guarapuava, Ponta Grossa, Mafra, Brasilândia, Luiziânia), (Campo Mourão, Cascavel, Palotina, Luiziânia), \\
& (Mandaguaçu, Palotina), (Luiziânia, Mandaguaçu), (Brasilândia, Mandaguaçu), (Campo Mourão, Mandaguaçu), \\
& (Cambará), (Castro), (Londrina). \\
\hline (Cascavel, Ponta Grossa, Mangueirinha), (Guarapuava, Mangueirinha, Campo Mourão), (Cascavel, Londrina), \\
\end{tabular}

${ }^{(1)}$ Os ambientes incluídos entre parênteses apresentaram interação linhagem x local não-significativa, a 5\% de probabilidade pelo teste F. 
Tabela 8. Estratificação ambiental, com base na discriminação fenotípica relacionada à produtividade de grãos, de linhagens de soja transgênica do grupo de maturação semiprecoce, no período de 2001/2002 a 2004/2005, em diversos locais do Paraná(1).

\begin{tabular}{|c|c|}
\hline Ano de avaliação & Conjunto de ambientes \\
\hline $2001 / 2002$ & $\begin{array}{l}\text { (Campo Mourão, Guarapuava, Ponta Grossa, Cascavel, Luiziânia, Cambará), (Castro, Palotina, Londrina, Cambará), } \\
\text { (Campo Mourão, Mafra), (Cascavel, Mandaguaçu), (Guarapuava, Mafra), (Mandaguaçu, Ponta Grossa), (Luiziânia, } \\
\text { Palotina), (Londrina, Ponta Grossa), (Campo Mourão, Londrina), (Cascavel, Castro), (Cascavel, Palotina), (Londrina, } \\
\text { Mandaguaçu), (Mangueirinha), (Pedrinhas). }\end{array}$ \\
\hline $2002 / 2003$ & $\begin{array}{l}\text { (Cascavel, Pedrinhas, Campo Mourão, Luiziânia, Guarapuava, Mafra, Cambará, Palotina, Castro), (Cascavel, Ponta } \\
\text { Grossa, Mandaguaçu), (Palotina, Ponta Grossa), (Cambará, Mangueirinha), (Londrina, Pedrinhas), (Mafra, } \\
\text { Mandaguaçu), (Mandaguaçu, Pedrinhas), (Mangueirinha, Palotina), (Mafra, Ponta Grossa), (Brasilândia, Pedrinhas), } \\
\text { (Brasilândia, Campo Mourão), (Cambará, Ponta Grossa), (Cascavel, Mangueirinha), (Tibagi). }\end{array}$ \\
\hline $2003 / 2004$ & $\begin{array}{l}\text { (Campo Mourão, Cascavel, Brasilândia, Mandaguaçu), (Mangueirinha, Ponta Grossa, Mafra, Guarapuava, } \\
\text { Mandaguaçu, Palotina, Luiziânia, Cambará), (Castro, Guarapuava, Campo Mourão), (Castro, Palotina), (Castro, } \\
\text { Mangueirinha), (Castro, Mandaguaçu), (Londrina, Mangueirinha), (Cascavel, Guarapuava). }\end{array}$ \\
\hline $2004 / 2005$ & $\begin{array}{l}\text { (Cascavel, Mangueirinha, Ponta Grossa), (Mafra, Ponta Grossa), (Campo Mourão, Ponta Grossa), (Cascavel, Mafra), } \\
\text { (Londrina, Mangueirinha), (Guarapuava, Londrina), (Brasilândia), (Palotina). }\end{array}$ \\
\hline
\end{tabular}

${ }^{(1)}$ Os ambientes incluídos entre parênteses apresentaram interação linhagem x local não-significativa, a 5\% de probabilidade pelo teste F.

\section{Conclusão}

1. No Estado do Paraná, não há diferença no desempenho produtivo entre as linhagens de soja convencional e de soja transgênica resistente ao glifosato, de diferentes ciclos de maturação, desenvolvidas pelo Programa de Melhoramento Genético da Embrapa Soja.

2. Os efeitos de local afetam mais intensamente a produtividade das linhagens da soja convencional e da soja transgênica resistente ao glifosato que os efeitos de ano, no Estado do Paraná.

3. A estratificação do Estado do Paraná não traz vantagens significativas e consistentes para a avaliação de linhagens de soja.

4. Não há clara indicação de locais que podem ser descartados para o teste das linhagens de soja.

\section{Agradecimentos}

À Financiadora de Estudos e Projetos e ao Conselho Nacional de Desenvolvimento Científico e Tecnológico, pelo custeio parcial da pesquisa e por concessão de bolsas.

\section{Referências}

ALLARD, R.W.; BRADSHAW, A.D. Implications of genotypeenvironmental interactions in applied plant breeding. Crop Science, v.4, p.503-507, 1964.

ALLIPRANDINI, L.F.; TOLEDO, J.F.F. de; FONSECA JÚNIOR, N.S.; ALMEIDA, L.A.; KIIHL, R.A. de S. Efeitos da interação genótipo x ambiente sobre a produtividade da soja no Estado do Paraná. Pesquisa Agropecuária Brasileira, v.29, p.1433-1444, 1994.
ARANTES, N.E. Interação genótipo $\mathbf{x}$ ambiente e estudo de alternativas para seleção de variedades de soja [Glycines max (L.) Merril], com base em testes regionais. 1979. 65p. Dissertação (Mestrado) - Universidade Federal de Viçosa, Viçosa.

CARVALHO, C.G.P. de; ARIAS, C.A.A.; TOLEDO, J.F.F. de; ALMEIDA, L.A. de; KIIHL, R.A. de S.; OLIVEIRA, M.F. de. Interação genótipo $\mathrm{x}$ ambiente no desempenho produtivo da soja no Paraná. Pesquisa Agropecuária Brasileira, v.37, p.989-1000, 2002.

CONAB. Central de informações agropecuárias. Disponível em: http://www.conab.gov.br/conabweb/index.php?PAG=131. Acesso em: 29 maio 2007.

LIN, C.S. Grouping genotypes by a cluster method directly related to genotype-environment interaction mean square. Theoretical and Applied Genetics, v.62, p.277-280, 1982.

MURAKAMI, D.M.; CRUZ, C.D. Proposal of methodologies for environment stratification and analysis of genotype adaptability. Crop Breeding and Applied Biotechnology, v.4, p.7-11, 2004.

PARANÁ. Secretaria da Agricultura e do Abastecimento. Oferta de sementes fortalece cultura da soja convencional. 2006. Disponível em: http://www.seab.pr.gov.br/modules/noticias/ article.php?storyid=2742. Acesso em: 29 maio 2007.

PIMENTEL-GOMES, F. Curso de estatística experimental. 11.ed. São Paulo: Nobel, 1985. 466p.

RAMALHO, M.A.P.; SANTOS, J.B.; ZIMMERMANN, M.J.O. Genética quantitativa em plantas autógamas: aplicações ao melhoramento do feijoeiro. Goiânia: UFG, 1993. 271p.

SAS INSTITUTE. SAS/STAT: user's guide: statistics. 5 $^{\text {th }}$ ed. Cary: SAS Institute, 1990. 1686p.

TOLEDO, J.F.F. de; CARVALHO, C.G.P. de; ARIAS, C.A.A.; ALMEIDA, L.A. de; BROGIN, R.L.; OLIVEIRA, M.F. de; MOREIRA, J.U.V.; RIBEIRO, A.S.; HIROMOTO, D.M. Genotype by environmental interaction on soybean yield in Mato Grosso State, Brazil. Pesquisa Agropecuária Brasileira, v.41, p.785-791, 2006.

Recebido em 28 de fevereiro de 2008 e aprovado em 5 de junho de 2008 